result, because the quantum-mechanical expression for interaction,

$$
g=\frac{\wp\left(\overrightarrow{r_{1}}\right) \wp\left(\overrightarrow{r_{2}}\right)}{S} \text {, }
$$

where $\varphi\left(\bar{r}_{1}\right)$ is potential acting on the first particle, and $S$ is the operator of the Schrödinger equation describing the interacting particles, reproduces the bilinear Fourier expansion of Green's function (Mercer's expansion), just as in the electrostatic case ${ }^{1}$.

There is another classical way of arriving at the Coulomb formula, when one starts, namely, not from the Laplace equation, but from $\Delta p-\ddot{p} / c^{2}=0$. The solution of the wave equation in functions of the four-dimensional distance $R=\sqrt{r^{2}+x_{4}^{2}}$ is $\varphi=1 / R^{2}$, which not only leads to the $r^{-1}$ law, upon integration over $x_{4}$, but also yields in the known manner retarded potentials, etc.

It may be of interest to get the similar fundamental invariant solution for the case of Proca equations. The solution of the equation $\Delta q-\ddot{q} / c^{2}-K_{0}^{2} \phi=0$ is found to be

$$
\varphi=\frac{K_{0}}{2} \frac{H_{1}^{1}\left(i K_{0} R\right)}{R},
$$

where $H_{1}^{1}$ denotes Hankel's function of the first kind and of the first order; this special solution has the right limit at $K_{0}=0$. Upon integrating this solution over the time $\left(x_{4}\right)$ in the static case where $r$ is a constant, for the point heavy 'charge' $g$, we obtain the required expression, $e^{-K_{0} r} / r$.

This method of reasoning not only gives a new dedustion of the Proca-Yukawa potential but can be used also for investigation of other special cases of classical 'mesodynamics', such as the analogue of retarded potentials, rate of emission of mesons by heavy particles, etc.

The most important problem is the construction of a classical model of the heavy mass by means of a mesonic field in the same manner as the electronic mass is built up from an electromagnetic field. IVe may mention here only three possible lines of attack on this problem : (1) the rough method of introduction of a radius $d$ of the heary particle. We get easily the following expressions for the self-energy of a heavy particle in the cases of a surface and a volume 2 'charged' heavy mass respectively :

$$
\begin{gathered}
E_{g}=\frac{1}{2} g^{2}\left(\frac{1}{d}+K_{0}\right) e^{-2 K_{0} d} ; \\
E_{v}=\frac{9}{4} \frac{g^{2}}{d^{6} K_{0}^{2}}\left\{\frac{2}{3} d^{3}-\frac{1}{K_{0}}\left(d^{2}-\frac{1}{K_{0}{ }^{2}}\right)-\frac{1}{K_{0}}\left(d+\frac{1}{K_{0}}\right)^{2} e^{-2 K_{0} d}\right\} .
\end{gathered}
$$

The empirical value $d \sim 10^{-13} \mathrm{~cm}$. gives too small an energy or heavy $b$ mass. (2) Dirac's method of direct omission of infinite terms in the expression for the electronic mass can be applied also in our case of heavy mass and mesonic field. Clearly, the nonelectromagnetic and non-mesonic fields respectively must be introduced here in some manner, as it is impossible to obtain the equations of motion from linear Maxwell and Proca equations. (3) The last mentioned extra-field playing the part of Poincaré's pressure can be associated with the non-linearities of the field. Following the Born-Infeld theory, say, as the most elaborated non-linear scheme, we can construct non-linear generalizations of Proca equations, which seem to be particularly appropriate in the case of neutral mesons.
We shall discuss in Journal of Physics ${ }^{3}$ the bearing of this non-linear limitation of quantum mechanics on cosmic ray phenomena, as well as other details of the above considerations.

D. Iwanenko.

State University,

Sverdlovsk, U.S.S.R.

1 Iwanenko, D., Sorc. Phys., 13, 172 (193s).

${ }^{2}$ Cf. Iwanenko, D., C.R. Acad. Sci. U.S.S.R., 21, 32 (1938).

3 A new journal of the Academy of Sciences, Moscow.

\section{A Pure Strain of Trichomonas eberthi in Tissue Culture}

IN May 1937 wo found in an explantate from a spontaneous leukxmia tumour in a fowl numerous specimens of Trichomonas eberthi. Sinco then wo have maintained the strain continuously on colonies of chicken fibroblasts. About 180 passages have been made up to the present time.

Tho flagellates are being cultured together with chicken fibroblast in hanging drop cultures according to the standard tissue culture technique of Carrel. For the culture medium, we used chicken plasma and chicken embryonic extract in 10 per cent dilution. As a rule the passages are mado every third day. To every passago a piece of an embryo chick's heart or a piece of a puro fibroblast culture is added as a substitute for the cells which are gradually destroyed.

$T$. eberthi multiplies intensively among the fibro. blasts and in the immediate neighbourhood of the tissue. They slowly liquefy the plasma coagulum, while spreading towards the periphery. After a period of forty-eight hours, the culture is pervaded and surrounded by a denso mass of active flagellates.

The presence of living cells is essential for the permanent culturo of this species in the plasmaembryonal coagulum. Several attempts wero mado to culturo $T$. eberthi in the samo medium in the presence of cells which had been previously killed by X-rays or heat, but all of them failed and the flagellates died out in the second passage. On the other hand, when $T$. eberth $i$ was cultivated in diluted embryonic extract alone, rich cultures wero obtained up to the twelfth passage during forty-five days.

E. Tenenbausi.

Department of Experimental Pathology

(Cancer Laboratories) and

Department of Parasitology, Hebrew University, Jerusalem.

May 5.

\section{"Introduction to Modern Genetics"}

WE are surprised at the review of Waddington's "Introduction to Modern Genetics" by Dr. F. W. Sansome in Nature of June 17, p. 1002. Wo aro at a loss to know what the reviewer means when he say's that Waddington's book does not "contain a balanced statement of modern genetics". It appears to us to be much the most satisfactory book on the subject written in recent years. Doubtless the scope could have been enlarged (for example, the treatment of adaptation and the mechanisms of speciation seems in places inadequate), and equally doubtless a book written ten years hence would adopt a different balance of subjects. Meanwhile, however, the most conspicuous feature of recent advance in genetics has 